\title{
SIMULTANEOUS OPERATION OF A TEST APPARATUS FILLED WITH LIQUID ARGON AS BUBBLE CHAMBER, CALORIMETER AND SCINTILLATION DETECTOR: OUTLOOK AND POSSIBLE APPLICATIONS
}

\author{
Gert G. HARIGEL
}

CERN, Geneva, Switzerland

Physics motivations for the use of argon as a new bubble chamber liquid are discussed. Results, obtained from a 2.71 argon detector in SPS and SC beams at CERN, comprise its track sensitivity to ionizing particles and to a laser beam in the bubble chamber mode, its use as a calorimeter through the collection of free charges in an electric field and the recording of the scintillation light produced by ionizing particles. Various interference phenomena during the simultaneous use of the hybrid properties, as well as purity requirements on the liquid are discussed. Furthermore, nitrogen and argon/nitrogen mixtures were investigated. Applications of our technique for neutrino experiments at $\mathrm{TeV}$ accelerators and as vertex detectors are briefly outlined. In-line holography, successfully tested in BEBC, could simplify the optical track recording and improve the resolution in very large detectors.

\section{Introduction}

Tests at CERN with argon, nitrogen and argon/nitrogen mixtures as new bubble chamber fluids were mainly motivated by the future need of cheap, relatively safe liquids in massive detectors for neutrino physics at $\mathrm{TeV}$ energies [1] for the study of baryon decay modes $[2,3]$ (if the proton or neutron decays) and to complement the line of existing pure cryogenic liquids, which can be used in one and the same (small) vertex bubble chamber for $A$-dependence measurements [4].

Argon is particularly interesting, because of its variety of properties: its track sensitivity during bubble chamber expansion to ionizing particles [5] and to a laser beam [6], the possibility of drifting free electric charges (electrons) over large distances [7] and the strong scintillation signal produced by ionizing particles when traversing the liquid [8]. These features can be employed simultaneously in combination with the well known advantages of the bubble chamber technique (homogeneous material, high resolution near the interaction vertex, curvature measurements in the magnetic field, energy range relation for slow particles, particle identification from ionization via bubble density), with the calorimetry by charge collection for very energetic electron or hadron showers, with the trigger from the fast scintillation pulse for the illumination of the bubble chamber photo when event rates are low and finally, with the laser induced bubble strings for sensitivity tests and fiducial systems.

The multitude of information obtained directly from liquid argon near the interaction itself is in many respects superior to the results generally acquired by the more complicated hybrid techniques used in combina- tion with hydrogen, deuterium or neon-hydrogen filled chambers, such as Track Sensitive Targets (TST), metal conversion plates or solid neon calorimeter inside the liquid; or by an External Muon Identifier (EMI), Internal Picket Fence (IPF), External Particle Identifier (EPI) or Identification of Secondary Particles by Ionisation Sampling (ISIS) outside the interaction volume.

\section{Test results with liquid argon}

More than 20 technical runs have been performed with a 2.71 argon bubble chamber, using a ${ }^{60} \mathrm{Co}$ source, a rf-separated beam from the SPS and mainly a 200 $\mathrm{MeV} / \mathrm{c}$ pion beam from SC at CERN. Results have been published on track formation $[5,6]$, on scintillation [7] and on calorimetry [8]. Therefore, we report here only some recent improvements of the test detector and interference effects between the electric field, the scintillation signal, the bubble formation and the influence of impurities in argon upon the above properties.

The general layout of the detector is shown in fig. 1 . A main modification has been made on the charge collection device inside the liquid: instead of a wire it consists now of three parallel plates; the middle one connected to the high voltage power supply and the charge sensitive preamplifier, the outer ones being connected to ground potential. This provides a fairly homogeneous electric field. Particles are injected into the gap parallel and close to the central plate. Only parts of the window facing the liquid are covered with an optically opaque wavelength shifter, in order to leave enough space for particle photography and track counting. The chamber, already mounted on its body, with the electric 


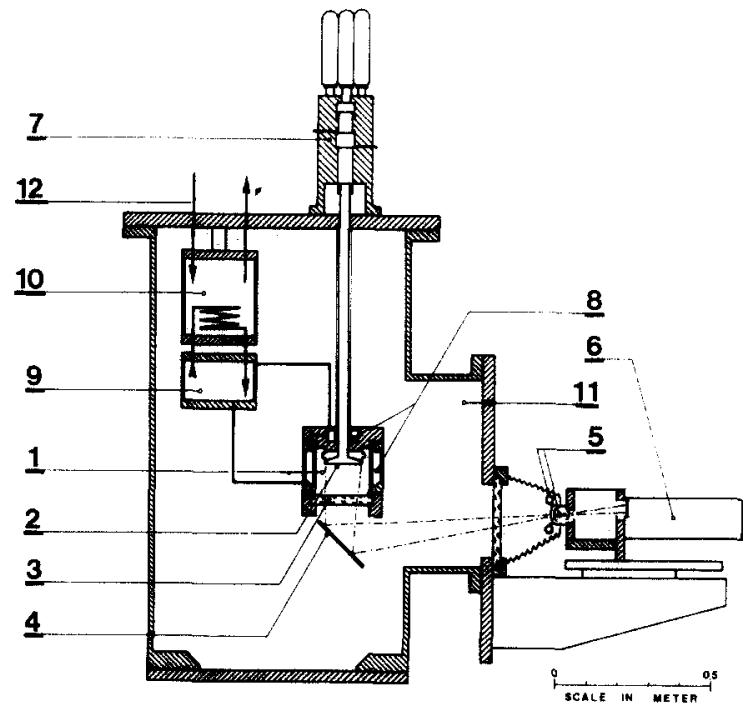

Fig. 1. Experimental argon bubble chamber: 1 - chamber body; 2 - expansion piston and Scotchlite; 3 - chamber window (for details see fig. 2); 4 - mirror; 5 - lens with annular flash; 6 - camera; 7 - expansion system; 8 - heat exchanger system; 9 - heat exchanger reservoir (in general liquid argon); 10 - refrigerant reservoir (in general nitrogen); 11 - vacuum tank; 12 - refrigeration (either dewar or refrigerator).

feedthrough is shown in fig. 2.

In a $200 \mathrm{MeV} / c$ pion beam, measurements have been made simultaneously of the scintillation and charge collection signals during the chamber's expansion. Fig. 3 shows a typical example of the amplitudes of these signals as a function of the applied voltage (distance

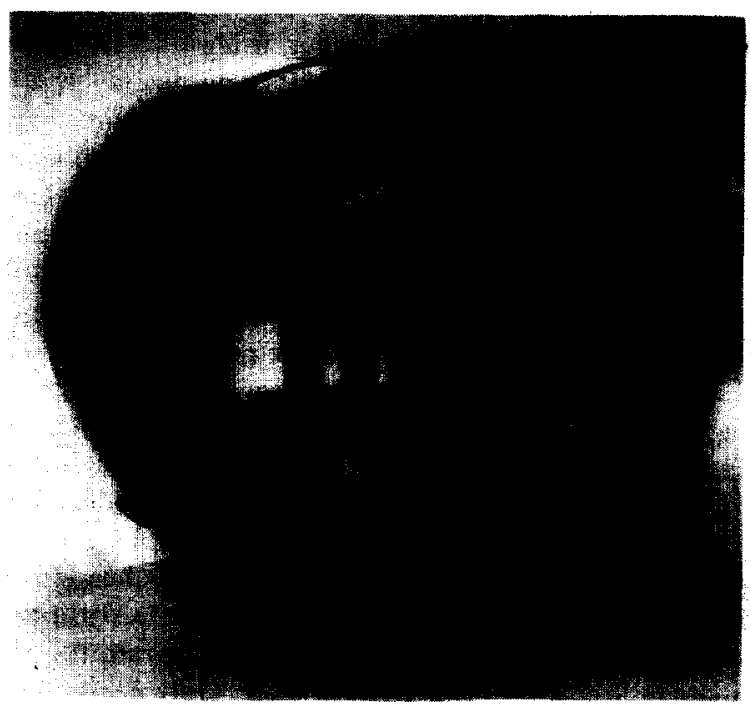

Fig. 2. Chamber vessel with window $(\varnothing=200 \mathrm{~mm}, d=70 \mathrm{~mm})$ and electric feeedthrough for high voltage supply and charge measurement. Distance between plates $25 \mathrm{~mm}$, gap between wavelength shifter $40 \mathrm{~mm}$.

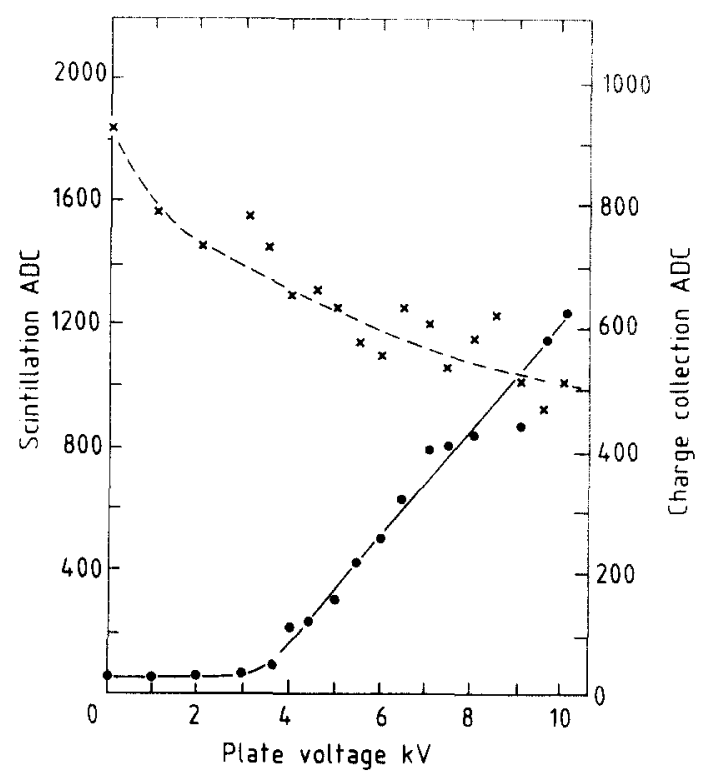

Fig. 3. Simultaneous measurement of charge collection ( $)$ and scintillation $(x)$ as a function of electric field, $\sim 110$ particles/pulse. Ordinates in arbitrary ADC units.

between the plates is $25 \mathrm{~mm}$ !). With increasing field strength more charges are collected, whereas the scintillation signal drops, as expected, due to the suppression of the recombination luminescence [9].

Controlled contamination of argon by injection of gaseous nitrogen showed the expected behaviour: a very rapid drop of charge collection efficiency and a much slower drop (almost linear in a semilogarithmic scale) of the scintillation signal by one order of magnitude between $\sim 0.1$ and $\sim 1500 \mathrm{ppm}$ nitrogen. No change in bubble density with increasing contamination was observed, indicating that nitrogen works as an acceptor from excited argon atoms $\mathrm{Ar}^{*}$, resulting in the emission of visible photons [10], which do not enhance bubble nucleation.

The application of electric fields up to $10 \mathrm{kV} / \mathrm{cm}$ perpendicular to the flight path of the particles did not alter to any measurable extent the bubble density along the tracks. This indicates that the bubble formation process - at least in argon - may be due to collisions of the second kind, rather than to electron-ion recombination or electrostatic repulsion. All the above mentioned effects will be investigated further in much more detail [11].

\section{Possible applications}

\subsection{Liquid argon in very large detectors}

It could be attractive to construct liquid argon bubble chambers for neutrino experiments at $\mathrm{TeV}$ accelera- 
Table 1

Tentative operating conditions of a multiliquid bubble chamber.

Chamber

Liquid

Atomic weight

Density

Radiation length

Temperature

Pressure

\section{$\mathrm{H}_{2}$}

1.0

0.06

$\sim 1000$

26

3.9

Cooling loop

Liquid

Temperature

Pressure

$\mathrm{H}_{2}$
25.5

3.5

Refrigerator

Liquid

Pressure

$\mathrm{H}_{2}$

$\geqq 1$

Bubble density ${ }^{\text {a) }}$

$\geqq 90$
$\mathrm{D}_{2}$

2.1

0.14

$\sim 900$

30

4.4
$\mathrm{N}_{2}$

14.0

0.60

65

113

17

$\mathrm{Ne}$
20.2
1.02
27
37
9

Ar

39.9

1.01

20

135

25
$\mathrm{Xe}$

131.3

2.3

3.9

252

26 $\mathrm{g} / \mathrm{cm}^{3}$
$\mathrm{~cm}$
$\mathrm{~K}$
$\mathrm{bar}$

a) For minimum ionizing particles; numbers depend on various parameters.

b) Pure neon has not yet been operated at this high temperature.

c) No published value available.

tors, where calorimetry and the scintillation signal could add valuable information to the track photography. Prior to any design, there remains at least one main technical question: should electrons be drifted over very large distances (about $100 \mathrm{~cm}$ ) radially towards the axis of a cylindrical chamber, essentially perpendicular to the bulk of tracks, giving timing information on the spread of the event, or should electrons drift longitudinally over smaller distances in track direction, where they would see a homogeneous electric field? The latter layout would give fewer problems with the purity of argon and high voltage power supply, but would give rise to more optical problems and an increase in parasitic boiling, due to the grid structure of the electrodes.

A giant liquid argon bubble chamber installed in a large underground laboratory could become an interesting device for the study of baryon decay modes: there the scintillation signal is an important feature of the detector, because it has to be used to trigger the bubble chamber illumination when a decay occurred $[2,3]$.

\subsection{Multiliquid bubble chamber}

The measurement of the $A$-dependence of various cross sections for incoming charged particles is an interesting subject to study at $\mathrm{TeV}$ energies. A small rapid cycling cryogenic bubble chamber could be installed in front of a multiparticle spectrometer. Our argon test chamber has the appropriate cooling circuit (see fig. 1) to allow, without any mechanical modifications, target fillings with pure hydrogen, deuterium, nitrogen, neon, argon and xenon (small admixtures of ethylene) or, if required, also with various neon/hydrogen or argon/ nitrogen mixtures. Tentative operating conditions (temperature and pressure) of the chamber liquids, of the cooling medium in the closed heat exchanger and of the cooling fluid in the refrigerator (dewar) are given in table 1 . Bubble densities are taken from various publications; bubble growth rates have acceptable values.

\subsection{In-line holography for large chambers}

The successful holographic recordings of cosmic ray induced bubble tracks in BEBC with an in-line technique [12] promise to open the way for the photography of giant bubble chambers with an improved optical resolution, compared with conventional bright-field photography. It may overcome some of the problems related to the use of Scotchlite, such as deposits of dust and parasitic boiling on its seams.

\section{References}

[1] G. Harigel, H. Kautzky, P. McIntyre and A. van Ginneken, Fermilab Proposal 601 (1978) unpublished.

[2] G.G. Harigel, these Proceedings (Adv. Detectors '83), p. 641.

[3] G. Harigel, A. Hervé and K. Winter, Nucl. Instr. and Meth. 216 (1983) 355.

[4] G. Harigel, in preparation.

[5] G. Harigel, G. Linser and F. Schenk, Nucl. Instr. and Meth. 187 (1981) 363. 
[6] G. Harigel, H.J. Hilke, G. Linser and F. Schenk, Nucl. Instr. and Meth. 188 (1981) 517.

[7] J.C. Berset, M. Burns, G. Harigel, J. Lindsay, G. Linser and F. Schenk, Nucl. Instr. and Meth. 203 (1982) 141.

[8] J.C. Berset, M. Burns, K. Geissler, G. Harigel, J. Lindsay, G. Linser and F. Schenk, Nucl. Instr. and Meth. 203 (1982) 133.

[9] S. Kubota, A. Nakamoto, T. Takahashi, T. Hamada, E. Shibamura, M. Miyajima, K. Masuda and T. Doke, Phys. Rev. B17 91978) 2762 .
[10] S. Himi, T. Takahashi, J.Z. Ruan and S. Kubota, Nucl. Instr. and Meth. 203 (1982) 152.

[11] G. Harigel, in preparation.

[12] H. Bjelkhagen, G. Harigel, F. Pouyat, W. Seidl, C. Baltay, M. Bregman, F. Eisler, M. Hibbs, A. Schaffer, R. Cence, E.B. Brucker and T.J. Hart, submitted to Nucl. Instr. and Meth. 